\title{
Análise da eficácia da ozonioterapia no tratamento da peri-implantite: uma revisão
}

\section{de escopo}

\author{
Analysis of the effectiveness of ozone therapy in the treatment of peri-implantitis: a scoping review \\ Análisis de la eficacia de la ozonoterapia en el tratamiento de la periimplantitis: una revisión del
}

alcance

Recebido: 23/12/2020 | Revisado: 29/12/2020 | Aceito: 13/01/2021 | Publicado: 15/01/2021

Camila Alvarenga da Silva

ORCID: https://orcid.org/0000-0003-0201-8020 Universidade de Brasília, Brasil

E-mail: camila.2008.alvarenga@hotmail.com

Thaís Salles Pereira

ORCID: https://orcid.org/0000-0003-2103-3568 Universidade de Brasília, Brasil

E-mail: thaissallespereia@gmail.com

Emily Caroline Silva Quirino

ORCID: https://orcid.org/0000-0001-5521-3247 Universidade de Brasília, Brasil

E-mail: emiilycaroliine1998@ hotmail.com

Isla Maria Pereira Ibiapina

ORCID: https://orcid.org/0000-0001-7066-9517 Universidade de Brasília, Brasil E-mail: islamaria@live.com

Aline Úrsula Rocha Fernandes

ORCID: https://orcid.org/ 0000-0001-8389-5628 Universidade de Brasília, Brasil E-mail: alineursula@unb.br

\begin{abstract}
Resumo
A peri-implantite é um processo inflamatório que afeta os tecidos ao redor de um implante osseointegrado em função, resultando na perda do osso de suporte. Uma técnica de limpeza aceitável deve ser capaz de desbridar e desintoxicar a superfície sem traumatizá-la. Sendo assim, o objetivo da presente revisão de escopo é avaliar se a ozonioterapia é eficaz no tratamento da peri-implantite. Para isso, o estudo utilizou o acrônimo PICOS. Foram incluídos estudos préclínicos in vivo em seres humanos, e clínicos que avaliaram a eficácia do tratamento da peri-implantite com ozônio. As buscas foram realizadas nas bases de dados PubMed, Embase, Scopus e Cochrane Library, e como literatura cinzenta foi realizada uma pesquisa no Google Scholar. Foi utilizada a estratégia de busca ""Ozone" AND ("PeriImplantitis" OR "Dental Implants" OR "Peri-implant")". O gerenciamento das referências foi realizado no software gerenciador de referência EndNote Basic. A ferramenta Cochrane foi utilizada para avaliar o risco de viés dos estudos que foram incluídos. Os resultados de todos os estudos foram descritos de forma narrativa. A presente revisão contou com a inclusão de três artigos. Foi possível constatar a redução significativa de todos os parâmetros clínicos analisados nos grupos ozônio, exceto a recessão de mucosa, que apresentou nenhuma ou mínima melhoria. Através dos resultados da presente revisão, foi possível concluir que a terapia de ozônio possui grande potencial para o tratamento da peri-implantite, apesar da alta heterogeneidade e a baixa qualidade dos estudos inseridos não permitirem uma evidência de alta qualidade.
\end{abstract}

Palavras-chave: Ozônio; Peri-implantite; Implantes dentários.

\begin{abstract}
Peri-implantitis is an inflammatory process that affects the tissues around an osseointegrated implant in function, resulting in the loss of the supporting bone. An acceptable cleaning technique must be able to debrid and detoxify the surface without traumatizing it. Therefore, the objective of the present scope review is to assess whether ozone therapy is effective in the treatment of peri-implantitis. For this, the study used the acronym PICOS. Pre-clinical in vivo studies in humans and clinical studies that evaluated the effectiveness of treating peri-implantitis with ozone were included. The searches were carried out in the PubMed, Embase, Scopus and Cochrane Library databases, and as a gray literature, a Google Scholar search was performed. The search strategy "“" Ozone "AND (" Peri-Implantitis "OR" Dental Implants "OR" Peri-implant ")" was used. The reference management was performed using the EndNote Basic reference manager software. The Cochrane tool was used to assess the risk of bias in the studies that were included. The results of all studies were described in a narrative manner. This review included three articles. It was
\end{abstract}


possible to see a significant reduction in all clinical parameters analyzed in the ozone group, except for the mucosal recession, which showed no or minimal improvement. Through the results of this review, it was possible to conclude that ozone therapy has great potential for the treatment of peri-implantitis, despite the high heterogeneity and the low quality of the inserted studies do not allow high quality evidence.

Keywords: Ozone; Peri-implantitis; Dental implants.

\section{Resumen}

La periimplantitis es un proceso inflamatorio que afecta a los tejidos alrededor de un implante osteointegrado en función, lo que resulta en la pérdida del hueso de soporte. Una técnica de limpieza aceptable debe ser capaz de desbridar y desintoxicar la superficie sin traumatizarla. Por tanto, el objetivo de la presente revisión del alcance es evaluar si la ozonoterapia es eficaz en el tratamiento de la periimplantitis. Para ello, el estudio utilizó el acrónimo PICOS. Se incluyeron estudios preclínicos in vivo en humanos y estudios clínicos que evaluaron la efectividad del tratamiento de la periimplantitis con ozono. Las búsquedas se realizaron en las bases de datos PubMed, Embase, Scopus y Cochrane Library, y como literatura gris, se realizó una búsqueda en Google Scholar. Se utilizó la estrategia de búsqueda "" Ozone "AND (" Peri-Implantitis "OR" Dental Implants "OR" Peri-implant ")". La gestión de referencias se realizó utilizando el software de gestión de referencias EndNote Basic. Se utilizó la herramienta Cochrane para evaluar el riesgo de sesgo en los estudios incluidos. Los resultados de todos los estudios se describieron de manera narrativa. Esta revisión incluyó tres artículos. Fue posible ver una reducción significativa en todos los parámetros clínicos analizados en el grupo de ozono, excepto la recesión de la mucosa, que mostró una mejora mínima o nula. A través de los resultados de la presente revisión, fue posible concluir que la ozonoterapia tiene un gran potencial para el tratamiento de la periimplantitis, a pesar de que la alta heterogeneidad y la baja calidad de los estudios insertados no permiten evidencia de alta calidad.

Palabras clave: Ozono; Periimplantitis; Implantes dentales.

\section{Introdução}

A reabilitação com implantes orais oferece algumas vantagens importantes sobre os tratamentos protéticos convencionais, porque a primeira conserva a estrutura do dente na dentição residual e reduz ou elimina a necessidade de próteses removíveis parciais ou completas (Busenlechner et al., 2014). A peri-implantite é, há muitos anos, um dos maiores desafios para os implantodontistas, afetando 16 a $28 \%$ dos pacientes implantados, em curto ou longo prazo (Lindhe et al., 2008). Esta alteração é definida como um processo inflamatório que afeta os tecidos ao redor de um implante osseointegrado em função, resultando na perda do osso de suporte (Madi et al., 2018).

O desbridamento mecânico com curetas está, em muitas pesquisas, associado às terapias adjuvantes e tem como objetivo aumentar a eficácia do tratamento não cirúrgico (Almeida et al., 2017). Apesar disso, Hauser-Gerspach et al. (2011) relataram que alguns desses instrumentos danificam a superfície do implante e causam contaminação metálica ou adesão de pó abrasivo à fixação do implante. Uma técnica de limpeza aceitável deve ser capaz de desbridar e desintoxicar a superfície sem traumatizá-la (Saffarpour et al., 2018). Sendo assim, se faz necessário um agente antimicrobiano eficiente, para auxiliar no tratamento da peri-implantite.

A clorexidina é um agente tópico comumente utilizado para o controle e prevenção da formação de biofilme, devido à sua alta substantividade, atividade bactericida e amplo espectro de ação (Liu et al., 2020). Schmidlin et al. (2012) mostraram que $80 \%$ dos dentistas, que participaram de sua pesquisa, utilizavam clorexidina no tratamento de peri-implantite. Porém, estudos mostraram que a terapia adjuvante com clorexidina pode não melhorar os resultados do tratamento não cirúrgico de doença peri-implantar (Liu et al., 2020). Em relação aos efeitos colaterais, sabe-se que a clorexidina pode causar descamação da mucosa, comprometimento da cicatrização de feridas e fixação de fibroblastos nas superfícies dentais, manchas nos dentes e alteração do paladar (Kshitish et al., 2010).

O ozônio, com sua poderosa propriedade de desinfecção, é considerado a substância perfeita para uso em procedimentos odontológicos, pois desinfeta os tecidos tratados e não deixa resíduos tóxicos, como produtos clorados (Razak et al., 2019). É comprovado que é eficaz contra vírus, fungos e bactérias Gram positivas e Gram negativas (Gupta et al., 2012). Além do efeito antimicrobiano, tem vários outros efeitos, como anti-hipóxico, imunomodulador, biossintético e analgésico 
(Saglam et al., 2020).

O ozônio é um gás instável e, para controlar a decomposição em oxigênio, ele pode ser associado a um veículo com propriedades aquosas para promover a conversão mais rapidamente ou a um veículo com propriedades mais viscosas, para retardar a conversão (Gupta et al., 2012). Sendo assim, tem sido usado clinicamente nas formas gasosa e aquosa, podendo ser dissolvido em água ou óleo (Saglam et al., 2020). Gás, óleo e água ozonizados podem ser aplicados nos sulcos, ou diretamente em abcessos ou mesmo em áreas de infecção crônica, para conter e eliminar as populações bacterianas (Domb et al., 2014).

Apesar das propriedades bacteriostáticas das substâncias ozonizadas serem comprovadas e alguns estudos mostrarem efeitos positivos na peri-implantite, ainda se tem dúvidas se é um tratamento eficaz. Sendo assim, o objetivo da presente revisão de escopo é avaliar se a ozonioterapia é eficaz no tratamento da peri-implantite.

\section{Metodologia}

A recomendação PRISMA-ScR foi adotada na pesquisa (Tricco et al. 2018).

- Estratégia de pesquisa

O estudo utilizou o acrônimo PICOT (P: pacientes com peri-implantite; I: ozônio; C: sem tratamento ou tratamento sem ozônio; O: periodonto submetido a tratamento para peri-implantite; T: Pré-clínicos in vivo em seres humanos, e clínicos). A pergunta a ser respondida é “A ozonioterapia é eficaz no tratamento da peri-implantite?"

As buscas foram realizadas por todo o mês de agosto de 2020, nas bases de dados PubMed, Embase, Scopus e Cochrane Library. Como literatura cinzenta, foi utilizado o Google Scholar. A estratégia de busca foi “ "Ozone" AND ("PeriImplantitis" OR "Dental Implants" OR "Peri-implant") ” como apresenta o Quadro 1.

Quadro 1. Estratégia de busca utilizada nas bases de dados.

\begin{tabular}{|c|c|}
\hline BASE & \multicolumn{1}{|c|}{ ESTRATÉGIA DE BUSCA } \\
\hline PubMed & "Ozone" AND ("Peri-Implantitis" OR "Dental Implants" OR "Peri-implant") \\
\hline Embase & "Ozone" AND ("Peri-Implantitis" OR "Dental Implants" OR "Peri-implant") \\
\hline Scopus & "Ozone" AND ("Peri-Implantitis" OR "Dental Implants" OR "Peri-implant") \\
\hline Cochrane Library & "Ozone" AND ("Peri-Implantitis" OR "Dental Implants" OR "Peri-implant") \\
\hline Google Scholar & "Ozone" AND ("Peri-Implantitis" OR "Dental Implants" OR "Peri-implant") \\
\hline
\end{tabular}

Fonte: Autores.

\section{- Critérios de inclusão e exclusão}

Os critérios de inclusão adotados foram: a) língua inglesa, portuguesa ou espanhola; b) estudos pré-clínicos in vivo em seres humanos, e clínicos; c) diagnóstico ou simulação de peri-implantite; d) tratamento realizado com ozônio; e) estudos que relatem o veículo ozonizado utilizado. Os critérios de exclusão adotados foram: a) descrição incompleta da metodologia e dos resultados do estudo e impossibilidade de obter tais informações; b) revisões, cartas, opiniões, capítulos de livros e abstracts de conferências; c) estudos in vitro, in vivo (animais), modelos epidemiológicos matemáticos e estudos com outros desenhos que não pré-clínicos in vivo em seres humanos, e clínicos; d) artigos que não se enquadrem nos critérios de inclusão. 


\section{- Identificação de estudos}

Após a busca, a primeira etapa foi a eliminação das duplicatas obtidas através da pesquisa, nas diferentes bases de dados, o gerenciamento das referências foi realizado no software gerenciador de referência EndNote Basic (Thomson Reuters, New York, NY). A segunda etapa foi a eliminação de artigos que não seguiam os critérios de inclusão e se encaixavam nos critérios de exclusão, pela leitura dos títulos e resumos. Quando esses não foram esclarecedores, foi realizada a inclusão do artigo para leitura na íntegra, para a última etapa de eliminação. A última etapa foi baseada na busca de informações completas, dentro dos objetivos do presente estudo.

\section{- Extração de dados}

Os dados extraídos foram alocados em 2 quadros (Quadros 2 e 3). O Quadro 2 trouxe informações gerais, como autores, ano de publicação, quantidade de pacientes, características do estudo, desenho do estudo, local do estudo e caracterização do protocolo de intervenção. O Quadro 3 apresentou os parâmetros clínicos da linha de base e do acompanhamento, além da conclusão de cada artigo incluído.

\section{- Análise e síntese de dados}

Todos os dados eram do tipo contínuos. Os resultados dos estudos foram descritos de forma narrativa.

\section{- Risco de viés}

O estudo utilizou a ferramenta Cochrane para avaliar o risco de viés dos estudos que foram incluídos. Este instrumento avalia os seguintes quesitos: geração de sequência aleatória (viés de seleção), ocultação de alocação (viés de seleção), cegamento de participantes e pessoal (viés de desempenho), cegamento da avaliação de resultados (viés de detecção), dados de resultados incompletos (tendência de atrito), relatórios seletivos (viés de relatórios) e outro viés. A qualidade metodológica abrangente de cada estudo foi julgada como de baixo risco se todos os sete domínios foram classificados como 'baixo risco de viés', como risco moderado, se pelo menos um domínio foi classificado como 'risco incerto de viés' e, alto risco, se um ou mais domínios foram avaliados como 'alto risco de viés' (Ren et al., 2016).

\section{- Avaliação GRADE para desenvolver recomendações}

Para avaliar a evidência, foi utilizado o método GRADE para fins de recomendações na prática clínica (Liu et al., 2015).

\section{Resultados}

A busca eletrônica nas bases de dados identificou 1.399 artigos. Após eliminação das duplicatas e análise dos títulos e resumos, de acordo com os critérios de inclusão e exclusão, consideramos 11 artigos como elegíveis, sendo realizada a leitura na íntegra dos 11 textos completos, dos quais, 8 foram excluídos por não serem o foco do estudo. Por fim, 3 artigos foram incluídos na presente revisão. A Figura 1 apresenta como foi realizada essa etapa. 
Research, Society and Development, v. 10, n. 1, e30210111465, 2021

(CC BY 4.0) | ISSN 2525-3409 | DOI: http://dx.doi.org/10.33448/rsd-v10i1.11465

Figura 1. Fluxograma das etapas de seleção dos artigos.

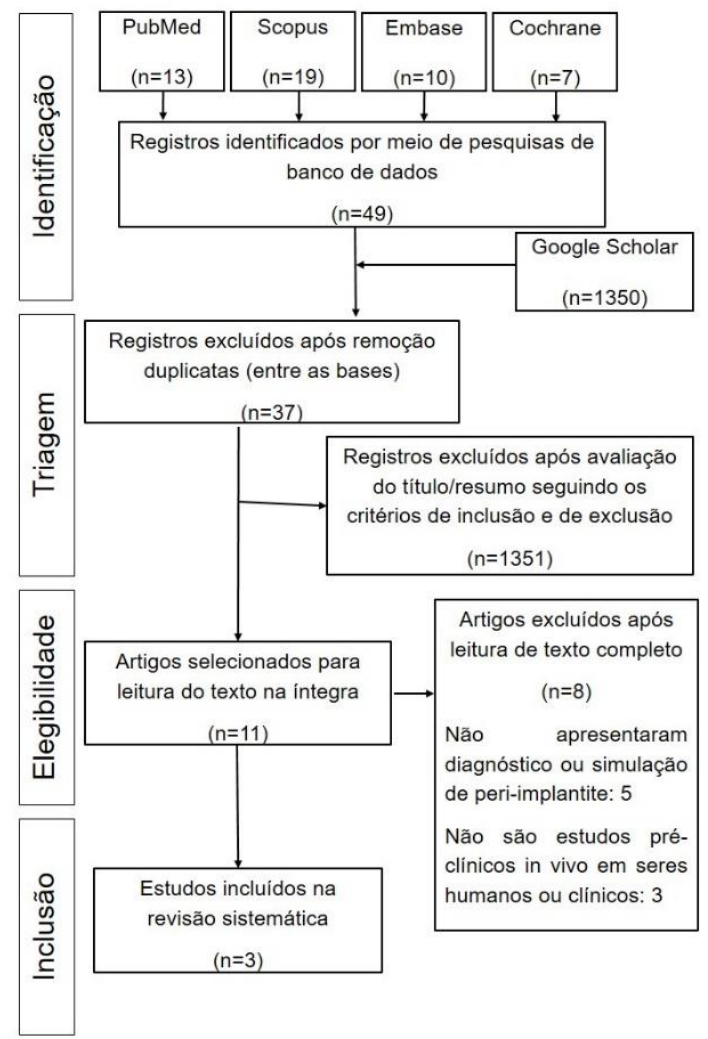

Fonte: Autores.

Os Quadros 2 e 3 mostram as principais características dos 3 estudos incluídos, sendo todos clínicos.

Quadro 2. Dados obtidos, a partir da análise dos artigos incluídos neste estudo.

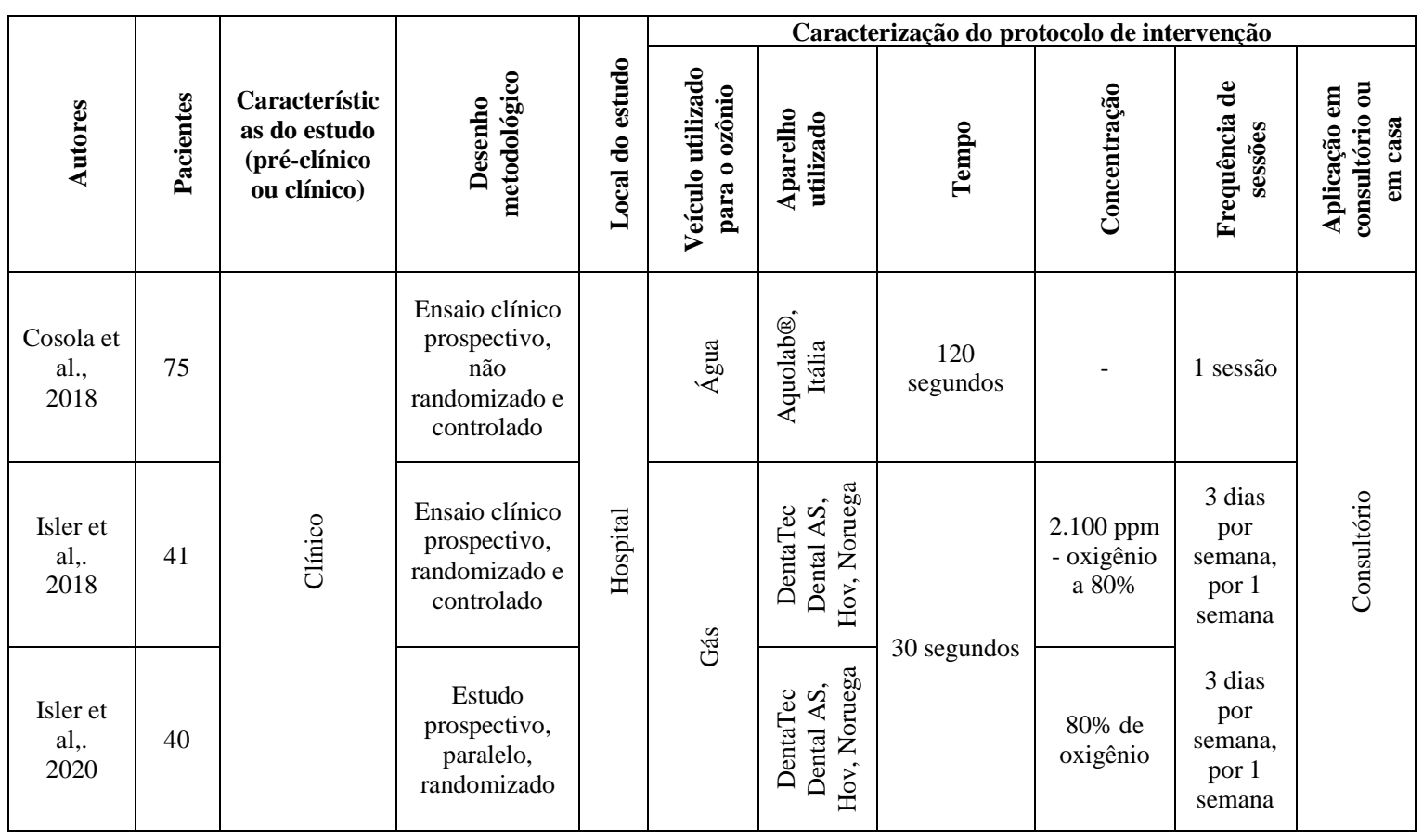

Fonte: Autores. 
Research, Society and Development, v. 10, n. 1, e30210111465, 2021

(CC BY 4.0) | ISSN 2525-3409 | DOI: http://dx.doi.org/10.33448/rsd-v10i1.11465

Quadro 3. Dados obtidos, a partir da análise dos artigos incluídos neste estudo.

\begin{tabular}{|c|c|c|c|c|c|c|c|c|c|c|c|}
\hline Autores & Pacientes & \multicolumn{9}{|c|}{ Resultados } & \multirow[t]{2}{*}{ Conclusão } \\
\hline & & & & IP & IG & SS (\%) & PS (mm) & NIC (mm) & $\mathrm{RM}(\mathrm{mm})$ & VDD (mm) & \\
\hline \multirow{3}{*}{$\begin{array}{l}\text { Cosola et al. } \\
\text { (2018) }\end{array}$} & \multirow{3}{*}{75} & Grupo 1 & $\begin{array}{c}\text { Linha de base } \\
\text { Acompanhamento de } 1 \text { mês } \\
\text { Acompanhamento de } 2 \text { meses }\end{array}$ & $\begin{array}{l}1,04 \pm 0,89 \\
0,56 \pm 0,77 \\
0,20 \pm 0,41\end{array}$ & - & $\begin{array}{l}0,80 \pm 0,76 \\
0,32 \pm 0,55 \\
0,12 \pm 0,33\end{array}$ & $\begin{array}{l}2,56 \pm 1,08 \\
2,14 \pm 1,02 \\
1,80 \pm 0,96\end{array}$ & - & $\begin{array}{l}0,42 \pm 0,59 \\
0,32 \pm 0,50 \\
0,30 \pm 0,50\end{array}$ & - & \multirow{3}{*}{$\begin{array}{c}\text { Todos os grupos mostraram } \\
\text { uma melhora significativa de } \\
\text { PS, IP e SS. Melhorias } \\
\text { clínicas mínimas foram } \\
\text { observadas também no RM, } \\
\text { mas não houve significância } \\
\text { estatística }\end{array}$} \\
\hline & & Grupo 2 & $\begin{array}{c}\text { Linha de base } \\
\text { Acompanhamento de } 1 \text { mês } \\
\text { Acompanhamento de } 2 \text { meses }\end{array}$ & $\begin{array}{l}1,72 \pm 1,10 \\
0,84 \pm 0,69 \\
0,52 \pm 0,65\end{array}$ & - & $\begin{array}{l}1,52 \pm 1,04 \\
0,80 \pm 0,71 \\
0,16 \pm 0,37\end{array}$ & $\begin{array}{l}3,36 \pm 1,11 \\
2,64 \pm 1,07 \\
2,48 \pm 1,08\end{array}$ & - & $\begin{array}{l}0,96 \pm 0,75 \\
0,76 \pm 0,68 \\
0,72 \pm 0,66\end{array}$ & - & \\
\hline & & Grupo 3 & $\begin{array}{c}\text { Linha de base } \\
\text { Acompanhamento de } 1 \text { mês } \\
\text { Acompanhamento de } 2 \text { meses }\end{array}$ & $\begin{array}{l}1,96 \pm 1,14 \\
1,40 \pm 0,87 \\
0,84 \pm 0,75\end{array}$ & - & $\begin{array}{l}1,84 \pm 0,85 \\
1,08 \pm 0,76 \\
1,00 \pm 1,94\end{array}$ & $\begin{array}{l}3,40 \pm 1,35 \\
2,96 \pm 1,06 \\
2,88 \pm 1,01\end{array}$ & - & $\begin{array}{l}1,38 \pm 0,87 \\
1,30 \pm 0,85 \\
1,28 \pm 0,82\end{array}$ & - & \\
\hline \multirow{2}{*}{$\begin{array}{l}\text { Isler et al. } \\
\text { (2018) }\end{array}$} & \multirow{2}{*}{41} & Grupo Ozônio & $\begin{array}{l}\text { Linha de base } \\
\text { Acompanhamento de } 3 \text { meses } \\
\text { Acompanhamento de } 6 \text { meses } \\
\text { Acompanhamento de } 12 \text { meses }\end{array}$ & $\begin{array}{l}1,21 \pm 0,57 \\
0,36 \pm 0,47 \\
0,27 \pm 0,38 \\
0,22 \pm 0,17\end{array}$ & $\begin{array}{c}1,15 \pm 0,53 \\
0,15 \pm 0,34 \\
0,025 \pm 0,1 \\
0,016 \pm 0,06\end{array}$ & $\begin{array}{c}96,6 \pm 10,85 \\
30 \pm 23,1 \\
21,6 \pm 27,6 \\
15,8 \pm 19,1\end{array}$ & $\begin{array}{c}6,27 \pm 1,42 \\
3,05 \pm 0,71 \\
2,69 \pm 0,81 \\
2,75 \pm 0,7\end{array}$ & $\begin{array}{l}6,39 \pm 1,23 \\
3,53 \pm 1,33 \\
3,23 \pm 1,48 \\
3,23 \pm 1,24\end{array}$ & $\begin{array}{c}0,12 \pm 0,14 \\
0,4 \pm 0,84 \\
0,54 \pm 0,88 \\
0,48 \pm 0,75\end{array}$ & $\begin{aligned} 4,63 & \pm 1,25 \\
& - \\
& - \\
2,54 & \pm 1,54\end{aligned}$ & \multirow{2}{*}{$\begin{array}{l}\text { No seguimento de } 12 \text { meses, } \\
\text { houve diferenças } \\
\text { significativas nos valores de } \\
\text { IP e GI entre os grupos, em } \\
\text { favor do grupo do ozônio. A } \\
\text { resolução da doença ocorreu } \\
\text { em } 50 \% \text { no grupo ozônio e } \\
36,6 \% \text { no grupo controle }\end{array}$} \\
\hline & & $\begin{array}{c}\text { Grupo Controle } \\
\text { (solução salina isolada) }\end{array}$ & $\begin{array}{l}\text { Linha de base } \\
\text { Acompanhamento de } 3 \text { meses } \\
\text { Acompanhamento de } 6 \text { meses } \\
\text { Acompanhamento de } 12 \text { meses }\end{array}$ & \begin{tabular}{l|}
$0,96 \pm 0,63$ \\
$0,63 \pm 0,64$ \\
$0,38 \pm 0,33$ \\
$0,49 \pm 0,27$
\end{tabular} & $\begin{array}{l}1,05 \pm 0,62 \\
0,29 \pm 0,62 \\
0,06 \pm 0,19 \\
0,07 \pm 0,14 \\
\end{array}$ & $\begin{array}{c}97,5 \pm 10,06 \\
28,33 \pm 30,06 \\
24,1 \pm 26,6 \\
25 \pm 21,7 \\
\end{array}$ & $\begin{array}{c}5,73 \pm 1,11 \\
3,44 \pm 1,3 \\
3,08 \pm 0,97 \\
3,34 \pm 0,85 \\
\end{array}$ & $\begin{array}{c}5,89 \pm 1,23 \\
3,84 \pm 1,6 \\
3,64 \pm 1,44 \\
3,91 \pm 1,36 \\
\end{array}$ & $\begin{array}{l}0,25 \pm 0,42 \\
0,46 \pm 0,57 \\
0,52 \pm 0,63 \\
0,55 \pm 0,64\end{array}$ & $\begin{aligned} 4,23 & \pm 1,46 \\
& - \\
& - \\
3,02 & \pm 1,57\end{aligned}$ & \\
\hline \multirow{2}{*}{$\begin{array}{l}\text { Isler et al. } \\
(2020)\end{array}$} & \multirow{2}{*}{40} & $\begin{array}{c}\text { Grupo } \\
\text { Ozônio }\end{array}$ & $\begin{array}{c}\text { Linha de base } \\
\text { Acompanhamento de } 6 \text { meses }\end{array}$ & $\begin{array}{l}1,14 \pm 0,19 \\
0,43 \pm 0,48\end{array}$ & $\begin{array}{l}1,27 \pm 0,57 \\
0,10 \pm 0,16\end{array}$ & $\begin{array}{l}93,75 \pm 11,30 \\
21,25 \pm 28,45\end{array}$ & $\begin{array}{l}6,75 \pm 1,00 \\
3,18 \pm 1,10\end{array}$ & $\begin{array}{l}6,83 \pm 1,04 \\
3,35 \pm 1,12\end{array}$ & - & $\begin{array}{l}3,39 \pm 0,92 \\
1,24 \pm 0,60\end{array}$ & \multirow{2}{*}{$\begin{array}{l}\text { Diferença estatisticamente } \\
\text { significante foi observada } \\
\text { entre os grupos, apenas para } \\
\text { os valores médios de NIC a } \\
\text { favor do grupo do ozônio }\end{array}$} \\
\hline & & $\begin{array}{c}\text { Grupo Controle } \\
\text { (solução salina isolada) }\end{array}$ & $\begin{array}{l}\text { Linha de base } \\
\text { Acompanhamento de } 6 \text { meses }\end{array}$ & $\begin{array}{l}1,16 \pm 0,32 \\
0,39 \pm 0,41\end{array}$ & $\begin{array}{l}1,18 \pm 0,51 \\
0,25 \pm 0,35\end{array}$ & $\begin{array}{c}95,83 \pm 9,73 \\
20,83 \pm 20,87\end{array}$ & $\begin{array}{l}6,37 \pm 0,91 \\
3,94 \pm 0,93\end{array}$ & $\begin{array}{l}6,43 \pm 1,00 \\
4,16 \pm 0,63\end{array}$ & - & $\begin{array}{l}3,23 \pm 0,73 \\
2,25 \pm 0,99\end{array}$ & \\
\hline
\end{tabular}

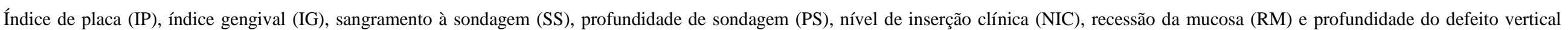
(VDD). Fonte: Autores. 
Dentro dos estudos incluídos, um total de 156 indivíduos, com diagnóstico de peri-implantite, em ao menos um implante dentário, foram avaliados. Para diagnóstico e análise do tratamento, foram verificados parâmetros clínicos, como índice de placa (IP), índice gengival (IG), sangramento à sondagem (SS), profundidade de sondagem (PS), recessão da mucosa (RM) e nível de inserção clínica (NIC).

Cosola et al. (2018) utilizaram um protocolo clínico para tratamento, sem associação cirúrgica, onde foi realizado desbridamento ultrassônico, polimento e enxágue com água ozonizada por dispositivo profissional específico, por 1 minuto. Eles dividiram os participantes da pesquisa em 3 grupos, seguindo faixas etárias pré-definidas. Todos os grupos mostraram uma melhora significativa de PS, IP e SS. Melhorias clínicas mínimas foram observadas também no RM, mas não houve significância estatística.

Os estudos de Isler et al. $(2018 ; 2020)$ apresentaram protocolo de intervenção análogos, em cujo grupo controle foi realizado desbridamento mecânico submucoso, seguido por irrigação de bolsa com solução salina por 3 minutos, nos locais de peri-implantite, e no grupo do ozônio, o ozônio gasoso adjuvante foi aplicado associado à irrigação com solução salina. O grupo ozônio, além de receber ozônio gasoso no momento da cirurgia, também recebeu no $3^{\circ}$ e $5^{\circ}$ dias de pós-operatório. Ozônio gasoso utilizado foi com $80 \%$ de oxigênio, por 30 segundos por dia, 3 dias por semana, durante 1 semana. No primeiro estudo, Isler et al. (2018) referiram uma diferença significativa, detectada apenas nos valores de PS entre os grupos, no período de acompanhamento de 3 meses, em favor do grupo do ozônio ( $p<0,05$ ). A média de RM aumentou significativamente em 3, 6 e 12 meses após o tratamento ( $<<0,05)$ em ambos os grupos em comparação com a linha de base. No seguimento de 12 meses, houve diferenças significativas nos valores de IP e IG entre os grupos, em favor do grupo do ozônio $(\mathrm{p}<0,05)$. Embora tenha havido uma tendência para uma maior redução dos valores de SS no grupo de ozônio, nenhuma diferença significativa entre os grupos de tratamento foi observada, em nenhum momento. Por fim, os resultados mostraram melhorias clínica e radiograficamente significativas na terapia regenerativa cirúrgica da peri-implantite em combinação com a terapia com ozônio e que o sucesso do tratamento foi obtido em 50\% dos implantes do grupo do ozônio e 36,6\% dos implantes do grupo controle. Em segundo estudo, Isler et al. (2020) apresentaram, no acompanhamento de 6 meses, que ambos os grupos demonstraram valores médios comparáveis com relação aos valores de IP, GI, SS, PS e na medição da profundidade do defeito vertical; enquanto uma diferença estatisticamente significante foi observada entre os grupos, apenas para os valores médios de NIC, a favor do grupo do ozônio $(\mathrm{p}=0,026)$.

\section{- Avaliação da qualidade metodológica}

Como apresentado nos Quadros 4 e 5, dos três estudos clínicos avaliados, apenas um teve um 'baixo risco de viés', os outros dois estudos foram designados como um com 'risco incerto de viés' e o outro 'alto risco de viés'. Os dois pontos que mais influenciaram para a classificação dos artigos, como não sendo de baixo risco de viés, foram o cegamento de participantes e pessoal (viés de desempenho), e cegamento da avaliação de resultados (viés de detecção). No estudo de Cosola et al. (2018), não se assumiu um grupo controle, com base nisso, não há cegamento dos resultados. Enquanto no estudo de Isler et al. (2018), os autores não deixaram explícito o cegamento dos pacientes, quanto ao procedimento, e dos profissionais, na análise dos parâmetros clínicos.

Quadro 4. Resumo do risco de viés: Avaliação da qualidade metodológica dos ensaios clínicos incluídos
Quadro 5. Avaliação da qualidade metodológica dos ensaios clínicos incluídos, apresentada como porcentagens 


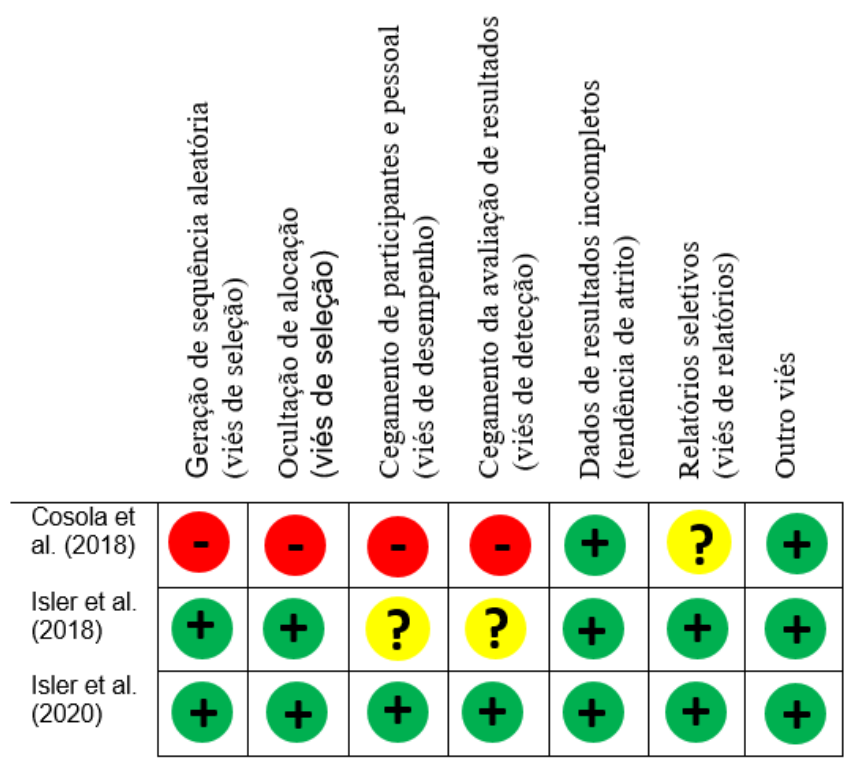

+: baixo risco de viés; -: alto risco de viés; ?: risco incerto.

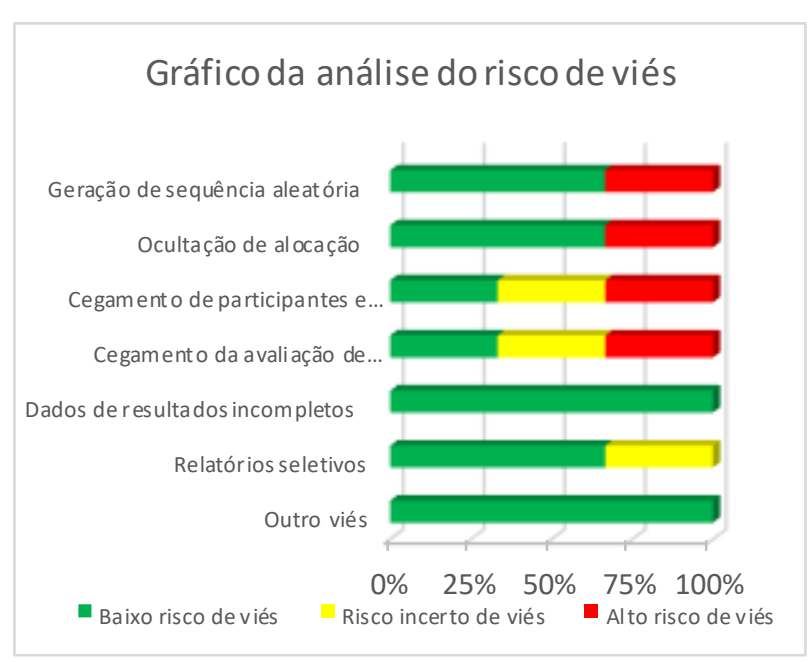

Fonte: Autores.

- Avaliação GRADE para desenvolver recomendações

A avaliação GRADE apresentou um nível de evidência baixo, isso ocorreu devido à alta heterogeneidade e a baixa qualidade dos estudos inseridos (Quadro 6).

Quadro 6. Quadro da avaliação GRADE.

\begin{tabular}{|c|c|c|c|c|c|c|c|c|c|c|}
\hline \multicolumn{7}{|c|}{ Certainty assessment } & \multicolumn{2}{|c|}{$\mathrm{Ne}$ de pacientes } & \multirow[b]{2}{*}{ Certainty } & \multirow[b]{2}{*}{ Importância } \\
\hline $\begin{array}{l}\text { Na dos } \\
\text { estudos }\end{array}$ & $\begin{array}{l}\text { Delineamento } \\
\text { do estudo }\end{array}$ & $\begin{array}{l}\text { Risco de } \\
\text { viés }\end{array}$ & Inconsistência & $\begin{array}{l}\begin{array}{c}\text { Evidencia } \\
\text { indireta }\end{array} \\
\end{array}$ & Imprecisăo & $\begin{array}{l}\text { Outras } \\
\text { considerạ̄oses }\end{array}$ & Ozônio & Sem ozônio & & \\
\hline \multicolumn{11}{|c|}{ Redução dos parâmetros clínicos } \\
\hline 3 & $\begin{array}{c}\text { ensaios } \\
\text { clinicos } \\
\text { randomizados }\end{array}$ & grave $^{\mathrm{a}}$ & não grave & năo grave & grave $^{b}$ & nenhum & $\begin{array}{l}115 / 156 \\
(73.7 \%)\end{array}$ & $\begin{array}{l}41 / 156 \\
(26.3 \%)\end{array}$ & $\bigoplus \bigoplus_{\text {BAIXA }}$ & CRITICO \\
\hline
\end{tabular}

Fonte: GRADEpro GDT.

\section{Discussão}

Os implantes dentários tornaram-se o padrão ouro para a reconstrução da dentição perdida. Décadas de investigação provaram que os implantes dentários são uma alternativa confiável, proporcionando função e estética, com sucesso de longo prazo (Amo et al., 2016). Mas, como relatado, a peri-implantite tem se apresentado como uma patologia que vem afetando a osseointegração e, consequentemente, reduzindo as taxas de sucesso dos implantes dentários.

Como terapia para a peri-implantite, existem algumas opções, entre elas opções cirúrgicas, não cirúrgicas e a combinação das duas terapias. Apesar dos inúmeros estudos em torno da peri-implantite, Suarez et al. (2013) afirmaram que não temos ainda protocolo clínico para o tratamento da peri-implantite, e revisaram algumas técnicas que vem sendo utilizadas, concluindo que são eficazes na eliminação da lesão inflamatória, mas que a osseointegração das superfícies previamente contaminadas tem sido difícil ou impossível de conseguir. Figuero et al. (2014) apresentaram que a terapia cirúrgica da periimplantite é indicada quando a terapia não cirúrgica falha em controlar as alterações inflamatórias. As três formas de abordagem foram avaliadas por Pommer et al. (2016), sendo o laser a opção não cirúrgica, e relataram que o sucesso do 
tratamento não diferiu entre a descontaminação a laser e/ou cirurgia de implantoplastia, mas que a abordagem com associação resultou em um maior atraso na perda do implante (6,5 anos), em comparação com a terapia a laser exclusiva (4,2 anos) ou cirurgia de implantoplastia ( 4,7 anos).

O objeto deste estudo foi avaliar se a ozonioterapia é eficaz no tratamento da peri-implantite. A terapia por ozônio vem sendo amplamente explorada na área da saúde. Nogales et al. (2008) apresentaram a indicação de seu uso para o tratamento de 260 diferentes patologias na medicina e odontologia. Apesar de pesquisas apresentarem suas propriedades favoráveis para o uso na clínica, ainda assim, poucos estudos e ausência de consenso permanecem sobre o uso em algumas patologias odontológicas (Nogales et al., 2008, Saini et al., 2011, Gupta et al., 2012, Razak et al., 2019).

Existem várias ações conhecidas do ozônio no corpo humano, como imunoestimulante e analgésico, anti-hipóxico e desintoxicante, antimicrobiano, bioenergético e biossintético, sendo que o ozônio em alta concentração causa efeito imunodepressor enquanto em sua baixa concentração causa efeito imunoestimulante (Gupta et al., 2012).

O gás ozônio tem um alto potencial de oxidação, sendo 1,5 vezes maior do que o cloreto, quando usado como agente antimicrobiano contra bactérias, vírus, fungos e protozoários, além da capacidade de estimular a circulação sanguínea e a resposta imunológica (Nogales et al., 2008). Saini et al. (2011) apresentaram ainda, que tratar pacientes com terapia de ozônio diminui o tempo de tratamento com uma grande variação e erradica a contagem bacteriana mais especificamente, o tratamento é indolor, quando realizada a aplicação tópica, e aumenta a tolerabilidade e satisfação dos pacientes com o mínimo de efeitos adversos. As complicações causadas pela terapia com ozônio são infrequentes em 0,0007 por aplicação, sendo os efeitos colaterais conhecidos epífora, irritação respiratória superior, rinite, tosse, dor de cabeça, náuseas ocasionais, vômitos, falta de ar, inchaço dos vasos sanguíneos, má circulação, problemas cardíacos e, às vezes, derrame (Gupta et al., 2012).

Na presente revisão, todos os estudos incluídos apresentaram redução significativa de todos os parâmetros clínicos analisados nos grupos ozônio (Quadro 3), exceto a recessão de mucosa, que apresentou nenhuma ou mínima melhoria, como apresentado no Quadro 3.Vale ressaltar, ainda, que nenhum dos estudos avaliados apresentou efeito adverso causado pela ozonioterapia.

A literatura (Valacchi \& Bocci, 1999, Traina, 2008, Hauser-Gerspach et al., 2011) apresenta, ainda, uma diferença gradual nos resultados, quando se aumenta a dose, sendo assim, o efeito aparentemente é dose dependente. Isto exige estudos que busquem testar o máximo de protocolos clínicos, a fim de concluir qual a melhor dose segura para o uso em periimplantite, respeitando sempre as concentrações potencialmente tóxicas. Bocci (2006) apresentou, em seu estudo, que, para empregar o ozônio na medicina, devemos evitar sua toxicidade, que somente pode ser controlada se operar com cautela, usando um gerador de ozônio preciso, equipado com fotômetro bem padronizado, que permita determinar o ozônio concentração em tempo real, coletando um volume de gás preciso com uma concentração de ozônio definida, além disso, devese saber a dose ideal para obter um efeito terapêutico sem toxicidade.

Vale ressaltar ainda, que o ozônio para fins médicos apresenta, normalmente, como produto final, uma mistura de gases que contém 95\% de oxigênio e 5\% de ozônio (Suh et al., 2019). Apesar disso, dois dos artigos utilizaram 80\% de oxigênio, devido às recomendações das instruções do fabricante do gerador de ozônio. É necessário se atentar as orientações do aparelho utilizado e buscar entender os motivos de tais orientações.

Uma limitação deste estudo foi a escassez de artigos encontrados na literatura, bem como a qualidade metodológica baixa de dois desses estudos clínicos (Quadros 3 e 4).

Em função da quantidade e qualidade metodológica dos estudos incluídos, não foi possível obter uma evidência de alta qualidade sobre a eficácia do uso da ozonioterapia como tratamento para peri-implantite (Quadro 6). Portanto, se faz necessária a realização de novos estudos in vitro e in vivo, contemplando as diversas populações, que analisem protocolos com diferentes veículos ozonizados, tempos de aplicação, concentrações de ozônio e frequência de sessões, para que, assim, seja 
definido um protocolo clínico adequado para tratamento eficaz da peri-implantite.

\section{Conclusão}

Através dos resultados da presente revisão, foi possível concluir que a terapia de ozônio, com suas poderosas propriedades, tem grande potencial no tratamento da peri-implantite, apesar da alta heterogeneidade e a baixa qualidade dos estudos inseridos não permitirem uma evidência de alta qualidade. São necessários mais estudos para resultados mais conclusivos e definição de protocolos clínicos eficientes.

\section{Agradecimentos}

Agradeço à UnB, por todo apoio.

Este trabalho foi financiado pela Universidade de Brasília, como projeto de iniciação científica, pelo Edital PROIC 2019/2020

\section{Referências}

Almeida, J. M. et al. (2017). Effectiveness of Mechanical Debridement Combined With Adjunctive Therapies for Nonsurgical Treatment of Periimplantitis. Implant Dentistry, 26 (1), 137-144. http://dx.doi.org/10.1097/id.0000000000000469.

Amo, F. S. D. et al. (2016). Non-Surgical Therapy for Peri-Implant Diseases: a systematic review. Journal Of Oral And Maxillofacial Research, 7 (3), 1-14. http://dx.doi.org/10.5037/jomr.2016.7313.

Bocci, V. A. (2006) Scientific and Medical Aspects of Ozone Therapy. State of the Art. Archives Of Medical Research, 37 (4), 425-435. http://dx.doi.org/10.1016/j.arcmed.2005.08.006.

Busenlechner, D. et al. (2014) Long-term implant success at the Academy for Oral Implantology: 8-year follow-up and risk factor analysis. Journal Of Periodontal \& Implant Science, 44 (3), 102-108. http://dx.doi.org/10.5051/jpis.2014.44.3.102.

Cosola, S. et al. (2018) Motivation of Patients to use Interdental Toothbrushes for the Domiciliary Management of Peri-Implants Health: different compliance according to the age. Dental Health: Current Research, 4 (2), 1-6. http://dx.doi.org/10.4172/2470-0886.1000132

Domb, W. (2014) Ozone Therapy in Dentistry. Interventional Neuroradiology, 20 (5), 632-636. https://doi.org/10.15274/INR-2014-10083.

Figuero, E. et al. (2000) Management of peri-implant mucositis and peri-implantitis. Periodontology 2000 , 66 (1), 255-273. http://dx.doi.org/10.1111/prd.12049.

Gupta, G. (2012) Ozone therapy in periodontics. J Med Life. 22 (5), 59-67. https://www.researchgate.net/publication/224934009_Oz one_therapy_in_periodontics

Hauser-gerspach, I. et al. (2011). Influence of gaseous ozone in peri-implantitis: bactericidal efficacy and cellular response. an in vitro study using titanium and zirconia. Clinical Oral Investigations, 16 (4), 1049-1059. http://dx.doi.org/10.1007/s00784-011-0603-2.

Isler, S. C. et al. (2020). The effects of decontamination methods of dental implant surface on cytokine expression analysis in the reconstructive surgical treatment of peri-implantitis. Odontology, 1-11. http://dx.doi.org/10.1007/s10266-020-00520-0.

Isler, S. C. et al. (2018). The effects of ozone therapy as an adjunct to the surgical treatment of peri-implantitis. Journal Of Periodontal \& Implant Science, 48 (3), 136-151. http://dx.doi.org/10.5051/jpis.2018.48.3.136.

Kshitish, D. \& Laxman, V. (2010). The use of ozonated water and $0.2 \%$ chlorhexidine in the treatment of periodontitis patients: a clinical and microbiologic study. Indian Journal Of Dental Research, 21 (3), 341-348. http://dx.doi.org/10.4103/0970-9290.70796.

Lindhe, J. \& Meyle, J. (2008). Peri-implant diseases: consensus report of the sixth european workshop on periodontology. Journal Of Clinical Periodontology, 35, 282-285. http://dx.doi.org/10.1111/j.1600-051x.2008.01283.x.

Liu, J. et al. (2015). Ozone therapy for treating foot ulcers in people with diabetes. Cochrane Database Of Systematic Reviews, 10, 1-35. http://dx.doi.org/10.1002/14651858.cd008474.pub2.

Liu, S. et al. (2020). chlorhexidine improve outcomes in non-surgical management of peri-implant mucositis or peri-implantitis?: a systematic review and meta-analysis. Medicina Oral Patología Oral y Cirugia Bucal, 25 (5), 608-615. http://dx.doi.org/10.4317/medoral.23633.

Madi, M. et al. (2018). Re-osseointegration of Dental Implants After Periimplantitis Treatments. Implant Dentistry, 27 (1), 101-110. http://dx.doi.org/10.1097/id.0000000000000712.

Nogales, C. G. et al. (2008). Ozone therapy in medicine and dentistry. J Contemp Dent Pract. 9 (4), 75-84. http://dx.doi.org/10.5005/jcdp-9-4-75. 
Research, Society and Development, v. 10, n. 1, e30210111465, 2021

(CC BY 4.0) | ISSN 2525-3409 | DOI: http://dx.doi.org/10.33448/rsd-v10i1.11465

Pommer, B. et al. (2016). Periimplantitis Treatment. Implant Dentistry, 25 (5), 646-649. http://dx.doi.org/10.1097/id.0000000000000461.

Razak, F. A. et al. (2019). Oxidizing Effect of Ozonated-Water on Microbial Balance in the Oral Ecosystem. Journal Of The College Of Physicians And Surgeons Pakistan, 29 (4), 387- 389. http://dx.doi.org/10.29271/jcpsp.2019.04.387.

Ren, C. et al. (2016). The effectiveness of low-level laser therapy as an adjunct to non-surgical periodontal treatment: a meta-analysis. Journal Of Periodontal Research, 52 (1), 8-20. http://dx.doi.org/10.1111/jre.12361.

Saffarpour, A. et al. (2018). Microstructural Evaluation of Contaminated Implant Surface Treated by Laser, Photodynamic Therapy, and Chlorhexidine $2 \%$. The International Journal Of Oral \& Maxillofacial Implants, 33 (5), 1019-1026. http://dx.doi.org/10.11607/jomi.6325.

Saglam, E. (2020). Evaluation of the effect of topical and systemic ozone application in periodontitis: an experimental study in rats. Journal Of Applied Oral Science, 28, 1-8. http://dx.doi.org/10.1590/1678-7757-2019-0140.

Saini, R. (2011). Ozone therapy in dentistry: a strategic review. Journal Of Natural Science, Biology And Medicine, 2 (2), 151-153. http://dx.doi.org/10.4103/0976-9668.92318.

Schmidlin, P. R. et al. (2012). Peri-implantitis prevalence and treatment in implantoriented private practices: A cross-sectional postal and Internet survey. Schweiz Monatsschr Zahnmed. 122 (12), 1136-1144. https://doi.org/10.5167/uzh-70578.

Suarez, F. et al. (2013). Implant Surface Detoxification. Implant Dentistry, 22 (5), 465-473. http://dx.doi.org/10.10++97/id.0b013e3182a2b8f4.

Suh, Y. et al. (2019). Clinical utility of ozone therapy in dental and oral medicine. Medical Gas Research, 9 (3), 163-167. http://dx.doi.org/10.4103/20459912.266997.

Traina, A. A. (2008). Efeitos biológicos do ozônio diluído em água na reparação tecidual de feridas dérmicas em ratos. Tese (Doutorado em Ciências Odontológicas - Universidade de São Paulo), São Paulo.

Tricco, A. C. et al. (2018). PRISMA extension for scoping reviews (PRISMA-ScR): checklist and explanation. Ann Intern Med, 169 (7), $467-473$. https://doi.org/10.7326/M18-0850.

Valacchi G, Bocci V. (1999). Studies on the biological effects of ozone: 10. Release of factors from ozonated humsn platelets. Mediat Inflammat; 8 (4-5), 205 209. https://doi.org/10.1080/09629359990360. 